\title{
Combining machine learning with dual-energy micro-CT to segment mineral phases
}

\author{
D. JENNINGS-GRAY ${ }^{1}$, S. BERG ${ }^{2}$, G. GARFI $^{1}$, S. KREVOR $^{1}$
}

${ }^{1}$ Imperial College London, Department of Earth Science and Engineering (daisy.jennings-gray16@imperial.ac.uk, g.garfi17@imperial.ac.uk,s.krevor@imperial.ac.uk)

${ }^{2}$ Shell Global Solutions International B.V., 2288 GS Rijswijk, The Netherlands (steffen.berg@shell.com)

Segmentation of micro-CT images of porous rock is an increasingly used method in petrophysics and hydrology. This research analyses the benefits of combining two individual segmentation techniques for mineral identification. Machine learning (ML) based image segmentation methods are used with dual energy micro-CT imagery [2] to produce mineral maps in a Berea sandstone. A Python algorithm based on WEKA [1] is adapted for dual energy imagery and to allow for 5 identification phases. Modalities are produced from applying filters to the two micro-CT images, then stacked and run through a Random Forest classifier. highlights the improvements made when a single energy ML segmentation is compared to a dual energy ML segmentation.
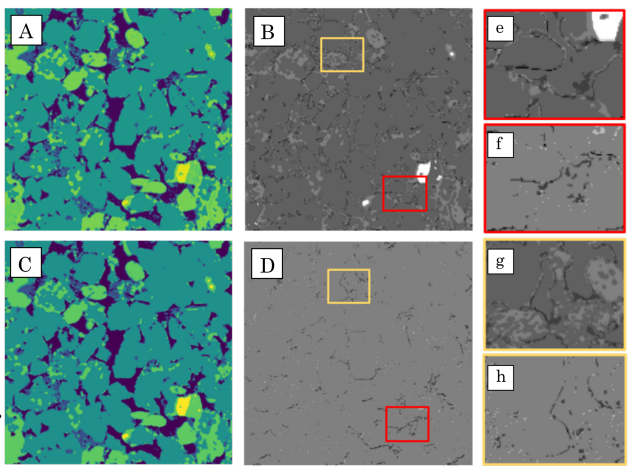

Figure 1: A single energy ML segmentation (A) compared to a dual energy ML segmentation (C). Difference images between the segmentations are generated (B, D) to emphasise regions of change $(\mathrm{E}-\mathrm{H})$, notably in the clay phase

Whilst the ML algorithm improves the accuracy of pore interconnectivites and grain angularity over other techniques, the single energy ML segmentation produces a film of erronous clay minerals around the edges of pores. Introducing a higher energy image modality to the stack of images reduces this error by providing better phase contrast.

[1] Arganda-Careras et al., (2017) Bioinformatics, 33(15), 2424-2426 [2] Lai (2015) Chemical Geology, 411, 260-273 
This abstract is too long to be accepted for publication.

Please revise it so that it fits into the column on one page. 